\title{
Analytic solution of wave loads on large vertical cylinder and its application
}

\author{
Fan Huiqu, Miao Quanming, Allan Ross Magee
}

\begin{abstract}
Based on Conformal Mapping method, the analytic solution of wave diffraction problem on the vertical cylinder with the cross section of arbitrary shape is proposed in this paper. The derivation of the solution is described in this paper and the results are compared with those of numerical methods. The good agreement shows that the analytic solution is correct and is applicable to non-circular cross section vertical cylinders. The model of scattering by a non-circular cross-section is a general basic mathematical problem. The techniques to handle the governing equation and boundary conditions in mapped plane proposed in this paper can provide reference for other similar Mathematical Physics problems.
\end{abstract}

Keywords-wave diffraction problem; conformal mapping method; wave load; Bessel function

\section{Introduction}

Wave loads calculation is the essential work in offshore platform design, only a few geometry shapes can be solved analytically, such as vertical or horizontal round cylinders $[1,2]$. With the help of conformal mapping technology, the ship's cross-section can be mapped to half-circle to get wave loads [3].

Non-circular section vertical cylinder's wave loads were investigated with dimensional analysis in early time [4]. During 1980s-1990s, Chinese scholars tried to use conformal mapping of rectangle section onto unit circle to get its wave potential. Zhao mapped rectangle onto unit circle and assumed the wave number remains the same value both in physical and mapped plane [5]. Huang assumed the incident wave will take the same shape in the mapped plane as in physical plane, only wave number is scaled $[6,7]$. Both Zhao and Huang show great work in their research. However, knowledge about the governing equation in the mapped plane and its features need be looked into deeply.

In this paper, the analytical method for vertical circular cylinder wave potential is followed. Using the series expansion of arbitrary cross-section onto the exterior of a unit circle, the wave potential equation in the mapped plane is derived and the solutions are classified with the boundary conditions for diffraction problem. Verification test cases show good consistency with BEM results.

Fan Huiqu

China Ship Scientific Research Center

PR. China

Miao Quanming

China Ship Scientific Research Center

PR. China

Allan Ross Magee

National University of Singapore

Singapore

\section{II.fundamental mathematical theory}

\section{A. Mathematical model of scattering by a circular cylinder}

This section describes the wave diffraction problem of a circular cylinder in constant water depth. The model in cylindrical coordinate mainly follows Mei's book [1,2], as the basis and starting point for non-circular section cylinder.

The fluid is assumed to be inviscid and incompressible and the motion is irrotational for water wave. Then the velocity $\overrightarrow{\mathrm{u}}$ can be expressed as the gradient of velocity potential $\Phi, \overrightarrow{\mathrm{u}}=\nabla \Phi$, and mass conservation requires the divergence of the velocity equal to zero, so $\Phi$ satisifies Laplace's equation:

$$
\nabla^{2} \Phi=0
$$

With the assumption of small wave amplitude, and definition of $\mathrm{z}$ axis directed vertically upwards and $\mathrm{z}=0$ in the plane of the undisturbed free surface. The wave can be linearized to satisfies the following conditions:

$$
\nabla^{2} \Phi=0,-h<z<0
$$

Sea bed boundary condition :

$$
\frac{\partial \Phi}{\partial \mathrm{n}}=0, \mathrm{z}=-\mathrm{h}
$$

Kinematic condition at free surface:

$$
\frac{\partial \zeta}{\partial \mathrm{t}}=\frac{\partial \Phi}{\partial \mathrm{z}}, \mathrm{z}=0
$$

Dynamic condition at free surface:

$$
\frac{\partial \Phi}{\partial \mathrm{t}}+\mathrm{g} \zeta+\mathrm{P}_{\mathrm{a}} / \rho=0, \mathrm{z}=0
$$

For constant water depth with $\mathrm{h}=$ cons $\mathrm{t}$, and timeharmonic motions of radian frequency $\omega$, time may be removed from the problem by writing:

$$
\Phi(x, y, z, t)=\phi(x, y, z) e^{-i \omega t}
$$

For 2-D problem, by separation of variables, $\phi=\phi(\mathrm{x}) \mathrm{f}(\mathrm{z})$, it is easy to get that

$$
\phi(x)=e^{ \pm i k x}
$$

And

$$
\begin{gathered}
\mathrm{f}^{\prime \prime}-\mathrm{k}^{2} \mathrm{f}=0,-\mathrm{h}<\mathrm{z}<0 \\
\mathrm{f}^{\prime}-\omega^{2} / \mathrm{gf}=0, \mathrm{z}=0 \\
\mathrm{f}^{\prime}=0, \mathrm{z}=-\mathrm{h}
\end{gathered}
$$


It is an eigenvalue problem of the Sturm-Liouville type. The solution is proportional to $\operatorname{coshk}(z+h)$, and the eigenvalue condition for $\mathrm{k}$ is the dispersion relation:

$$
\omega^{2} / \mathrm{g}=\mathrm{k} \tanh (\mathrm{kh})
$$

In the interval $[-\mathrm{h}, 0]$, any function can be represented by a Fourier series based on $\left\langle\mathrm{f}_{\mathrm{n}}\right\rangle$. Consequently, one can use the series

$$
\phi(x, z)=a_{0} f_{0}(z) e^{ \pm i k x}+\sum_{n=1}^{\infty} b_{n} f_{n}(z) e^{\mp k_{n} x}
$$

to represent the potential of a radiated or scattered wave. The first term corresponds to the propagating mode, while the series terms are only of local importance and are called the evanescent modes.

For 3-D problem, the Laplace equation in cylindrical coordinates is

$$
\frac{1}{r} \frac{\partial}{\partial r}\left(r \frac{\partial \phi}{\partial r}\right)+\frac{1}{r^{2}} \frac{\partial^{2} \phi}{\partial \theta^{2}}+\frac{\partial^{2} \phi}{\partial z^{2}}=0
$$

The eigensolution corresponding to the real and imaginary eigenvalues are:

$$
\begin{gathered}
\left\{\begin{array}{l}
H_{m}^{1}(k r) \\
H_{m}^{2}(k r)
\end{array}\right\}\left\{\begin{array}{l}
\cos (m \theta) \\
\sin (m \theta)
\end{array}\right\} f_{0}(z), m=0,1,2,3 \ldots \ldots \\
\left\{\begin{array}{l}
I_{m}(k r) \\
K_{m}(k r)
\end{array}\right\}\left\{\begin{array}{l}
\cos (m \theta) \\
\sin (m \theta)
\end{array}\right\} f_{n}(z), n=1,2,3 \ldots \ldots
\end{gathered}
$$

Where $\mathrm{H}_{\mathrm{m}}^{1}, \mathrm{H}_{\mathrm{m}}^{2}$ are the Hankel functions of the first and second kinds, and $\mathrm{I}_{\mathrm{m}}, \mathrm{K}_{\mathrm{m}}$ are the modified Bessel functions of the first and second kinds.

For wave-structure, if water depth is constant, an incident plane wave with time removed can be expressed as:

$$
\phi_{I}=e^{i k r \cos (\theta-\beta)} f_{0}(z)
$$

where $\beta$ is the heading angle.

If the scattering object has constant cross-section and extends throughout the water depth, the orthogonality of the functions $\left\langle\mathrm{f}_{\mathrm{n}}\right\rangle$ implies that the diffracted wave will also be proportional to $f_{0}(z)$, and only the propagating mode exists.

The potential for the problem can be thus decomposed as

$$
\phi(r, \theta, z)=\varphi(r, \theta) f_{0}(z)
$$

and since $\phi$ satisfies Laplace equation, then $\varphi$ satisfies Helmholtz equation :

$$
\nabla^{2} \varphi+k^{2} \varphi=0
$$

throughout the fluid domain.

For the scattering problem of an incident plane wave of wavenumber $k$ by a bottom-mounted vertical circular cylinder with $r=a$ standing in water of constant depth $\mathrm{h}$, the potential of the incident wave can be written as (with time and $\mathrm{f}_{0}(\mathrm{z})$ removed)

$\phi_{I}=e^{i k r \cos (\theta-\beta)}=\sum_{m=0}^{\infty} \epsilon_{m} i^{m} J_{m}(k r) \cos (m(\theta-\beta))$

where $\epsilon_{\mathrm{m}}$ is Neumann symbol, $\epsilon_{0}=1, \epsilon_{\mathrm{m}}=2(\mathrm{~m} \geq 1)$.

Since the scattered wave must behave like an out-going wave as $k r \rightarrow \infty$, the scattered wave can be written in the following form

$$
\phi_{S}=\sum_{m=0}^{\infty} a_{m} \epsilon_{m} i^{m} H_{m}^{1}(k r) \cos (m(\theta-\beta))
$$

where the complex coefficient $a_{m}$ are to be determined and the orthogonality of the functions in $\theta$ has been used to eliminate terms in $\sin (m \theta)$.

Application of the body boundary condition

$$
\frac{\partial \phi_{I}}{\partial n}=\frac{\partial \phi_{I}}{\partial r}=-\frac{\partial \phi_{S}}{\partial n}=-\frac{\partial \phi_{S}}{\partial r}(r=a)
$$

And by application of the orthogonality of the functions $\cos (m \theta)$ on $(0,2 \pi)$, we can solve for the unknowns $a_{m}$.

Thus, the total potential of the problem is

$$
\begin{gathered}
\phi_{D}=\phi_{I}+\phi_{S}= \\
\sum_{m=0}^{\infty} \epsilon_{m} i^{m}\left(J_{m}(k r)-\frac{J_{m}^{\prime}(k a)}{H_{m}^{1} k a} H_{m}^{1}(k r)\right) \operatorname{cosm}(\theta-\beta)
\end{gathered}
$$

Due to Wronskian relations satisfied by Bessel functions, it can be simplified as on a cylinderical surface:

$$
\phi_{D}=\phi_{I}+\phi_{S}=\sum_{m=0}^{\infty}\left(\frac{2 \epsilon_{m} i^{m+1}}{\pi k a H_{m}^{1^{\prime} k a}}\right) \cos m(\theta-\beta)
$$

\section{B. Basic of conformal mapping and some properties}

In planar irrotational flow analysis, by finding a proper conformal transformation, a complex 2-D boundary can be transferred to a simple one in the mapped plane. In the mapped plane, we apply simplified boundary conditions to get the complex potential, then the problem with complicated boundary in physical plane can be solved using parameters in the mapped plane. It is common to transfer the boundary in 
Proc. of the Seventh International Conference On Advances in Applied Science and Environmental Engineering - ASEE 2017. Copyright (C) Institute of Research Engineers and Doctors. All rights reserved.

ISBN: 978-1-63248-125-2 doi: 10.15224/ 978-1-63248-125-2-10

the physical plane onto circular or straight lines in the mapped plane $[8,9]$.

In complex analysis, the Riemann mapping theorem states: Let D be any simply connected domain in the plane other than the entire plane itself. Then there is a one-to-one analytic function that maps $D$ onto the open unit disk. Moreover, one can prescribe an arbitrary point of $D$ and a direction through that point which are to be mapped to the origin and the direction of the positive real axis, respectively. Under such restrictions, the mapping is unique.

Finding a suitable mapping which will simplify the boundary condition is essential for a given problem.

For complex potential of flow around a cylinder, if a mapping $z=f(\zeta)$ is found can map the given boundary to a circle, then the flow potential is easy to be obtained. To map the exterior of 2-D object onto the exterior of a circle and the external flow far away from the boundary keeps its direction and magnitude unchanged, which means the flow only changes around the mapped boundary and is unchanged far away. Thus, the map requires:

$\zeta \sim \mathrm{z}$, when $|\mathrm{z}| \rightarrow \infty$, and

$$
\frac{d \zeta}{d z}=1,|z| \rightarrow \infty
$$

So, if to take the map $z=f(\zeta)$ in the form of Laurent series away from origin, the one fulfill the requirements should be:

and

$$
z=\zeta+\sum_{n=1}^{\infty} \frac{c_{n}}{\zeta_{n}}
$$

$$
\zeta=z-\frac{c_{1}}{Z}+\sum_{n=2}^{\infty} \frac{c_{n}^{\prime}}{Z^{n}}
$$

where $c_{1}, c_{2} \ldots$ are complex variables, related to the shape of the objects, and $c_{n}^{\prime}$ may not necessary equals to $c_{n}$. In the equation, $c_{0}$ has been omitted by adjusting the orgin of $\zeta$ plane. And if the boundary in $\mathrm{z}$ plane mapped onto unit circle in $\zeta$ plane, the uniform mapping relation can be written as

$$
z=\omega(\zeta)=\sum_{n=0}^{\infty} a_{n} \zeta^{1-n}
$$

With complex coefficients, which can be determined with numerical methods. In this paper Melent'ev method is used for them [9].

For the mapping relation of (10), we have the following properties:

\section{1) The case of symmetric contours}

If the region $\mathbf{D}$ bounded by the curve $\Gamma$ has $\mathrm{p}$ axes of symmetry, the mapping function will hold the following form for exterior regions.

$$
z=\sum_{k=0}^{\infty} \alpha_{k} \zeta^{1-k p}
$$

which shows the non-zero terms only lie on $0, p, 2 p, 3 p .$. ones. The distribution of non-zero terms provides results justification references for symmetry cases.

\section{2) Mapping Parameters relationship}

The derivative of the mapping function is

$$
\frac{\partial z}{\partial \zeta}=\sum_{n=0}^{\infty}(1-n) a_{n} \zeta^{-n}
$$

If the mapping function is represented by polar coordinates $(r, \theta)$ both in physical and mapped plane, and applying implicit differentiation rule, we will get the following relationship of $\frac{\partial \mathrm{r}}{\partial \rho}, \frac{\partial \theta}{\partial \rho}, \frac{\partial \mathrm{r}}{\partial \psi}, \frac{\partial \theta}{\partial \psi}$.

The details are:

By writing:

$$
r e^{(i \theta)}=\sum_{n=0}^{\infty} a_{n}\left(\rho e^{i \psi}\right)^{1-n}
$$

and application of implicit differentiation rule, we have:

$$
\frac{\partial r}{\partial \rho}=e^{i(\psi-\theta)} \sum_{n=0}^{\infty} a_{n} \zeta^{-n}(1-n)=e^{i(\psi-\theta)} \frac{d z}{d \zeta}
$$

$$
\begin{aligned}
\frac{\partial \theta}{\partial \rho}=e^{i(\psi-\theta)} /(i r) & \sum_{n=0}^{\infty} a_{n}(1-n) \zeta^{-n}=e^{i(\psi-\theta)} \frac{d z}{d \zeta} /(i r) \\
= & -\frac{i}{r} \frac{\partial r}{\partial \rho}
\end{aligned}
$$

$$
\begin{aligned}
& \frac{\partial r}{\partial \psi}=i \rho e^{i(\psi-\theta)} \sum_{n=0}^{\infty} a_{n}(1-n) \zeta^{-n}=i \rho e^{i(\psi-\theta)} \frac{d z}{d \zeta}=i \rho \frac{\partial r}{\partial \rho} \\
& \frac{\partial \theta}{\partial \psi}=\rho e^{i(\psi-\theta)} / r \sum_{n=0}^{\infty} a_{n}(1-n) \zeta^{-n}=\frac{\rho}{r} e^{i(\psi-\theta)} \frac{d z}{d \zeta}=\frac{\rho}{r} \frac{\partial r}{\partial \rho}
\end{aligned}
$$

3) Resultant forces around the boundary

Say $\Gamma$ is the section boundary, then resultant force in physical plane can be obtained by:

$$
\begin{gathered}
F_{x}=-\int_{\Gamma} p(r, \theta) \cos \theta d s=-\int_{\Gamma} p(r, \theta) \cos \theta r d \theta \\
F_{y}=-\int_{\Gamma} p(r, \theta) \sin \theta d s=-\int_{\Gamma} p(r, \theta) \sin \theta r d \theta
\end{gathered}
$$

If $F_{x}$ is represented by parameters in the mapped plane, we have: 
Proc. of the Seventh International Conference On Advances in Applied Science and Environmental Engineering - ASEE 2017. Copyright (C) Institute of Research Engineers and Doctors. All rights reserved.

ISBN: 978-1-63248-125-2 doi: 10.15224/ 978-1-63248-125-2-10

$$
\begin{aligned}
& F_{x} \\
& =-\int_{0}^{2 \pi} p(r(1, \psi), \theta(1, \psi)) \cos (\theta(1, \psi)) r(1, \psi) d \theta(1, \psi)
\end{aligned}
$$

By Substituting

$$
d \theta(1, \psi)=\frac{e^{i(\psi-\theta(1, \psi))}}{r(1, \psi)} \frac{d z(1, \psi)}{d \zeta(1, \psi)} d \psi
$$

then the result is found to be

$$
\begin{gathered}
F_{x}=-\int_{0}^{2 \pi} p(r(1, \psi), \theta(1, \psi)) \cos (\theta(1, \psi)) \\
e^{i(\psi-\theta(1, \psi))} \frac{d z(1, \psi)}{d \zeta(1, \psi)} d \psi
\end{gathered}
$$

Similarly,

$$
\begin{gathered}
F_{y}=-\int_{0}^{2 \pi} p(r(1, \psi), \theta(1, \psi)) \sin (\theta(1, \psi)) \\
e^{i(\psi-\theta(1, \psi))} \frac{d z(1, \psi)}{d \zeta(1, \psi)} d \psi
\end{gathered}
$$

\section{Mathematical model of wave past cylinder with non- circular cross section}

\section{A. wave potential}

The foregoing shows that wave potential in horizontal plane follows Helmholtz equation. For non-circular section, there is no clear analytic solution for it, the usual way is to discretize the boundary $\Gamma$ into several segments and solve it with BEM.

In this paper, by combining the conformal transformation and cylinder wave scattering analysis in cylindrical system, the Helmholtz equation will be expanded with negative degree power series of the mapping function with simplified boundary conditions to get its series solution.

Let $\Gamma$ be the boundary of the object in physical plane $\mathrm{z}$, it's known from the Riemann mapping theorem there exists a mapping function which maps $\Gamma$ onto unit circle in the mapped plane $\zeta$. And then, Helmholtz equation in $\zeta$ plane is transformed to:

$$
\Delta \phi_{s}+\left[k\left|\frac{\partial z}{\partial \zeta}\right|\right]^{2} \phi_{s}=0
$$

Compared with the one in z plane, the First-order term's coefficient changes from $\mathrm{k}$ into $\mathrm{k}\left|\frac{\partial \mathrm{z}}{\partial \zeta}\right|$. If we let $\zeta=\rho \mathrm{e}^{(\mathrm{i} \psi)}$ represent the points in $\zeta$ plane with polar coordinates, around the unit circle $\frac{\partial \mathrm{z}}{\partial \zeta}$ will vary. It means that by conformal mapping, the wavenumber in the mapped plane varies from original $\mathrm{k}$ in $\mathrm{z}$ plane to $\mathrm{k}\left|\frac{\partial \mathrm{z}}{\partial \zeta}\right|$, and it is changing with the position of the points. That is the mathematical root of the complexity of the problem for non-circular section.

From (12), it is known that:

$$
\begin{aligned}
\left|\frac{\partial z}{\partial \zeta}\right|^{2}=\frac{\partial z}{\partial \zeta} \cdot \overline{\left(\frac{\partial z}{\partial \zeta}\right)} & =\left(\sum_{n=0}^{\infty} \frac{(1-n) a_{i}}{\zeta^{n}}\right) \cdot \overline{\left(\sum_{n=0}^{\infty} \frac{(1-n) a_{i}}{\zeta^{n}}\right)} \\
& =\left(\sum_{n=0}^{\infty} \frac{(1-n) a_{i}}{\zeta^{n}}\right) \cdot\left(\sum_{n=0}^{\infty} \frac{(1-n) \overline{a_{i}}}{\overline{\zeta^{n}}}\right)
\end{aligned}
$$

By substituting $\zeta=\rho \mathrm{e}^{(\mathrm{i} \psi)}$

$$
\begin{aligned}
\left|\frac{\partial z}{\partial \zeta}\right|^{2}=\left(\sum_{n=0}^{\infty} \frac{(1-n) a_{i}}{\left(\rho e^{(i \psi)}\right)^{n}}\right) \cdot\left(\sum_{n=0}^{\infty} \frac{(1-n) \overline{a_{i}}}{\overline{\left(\rho e^{(i \psi)}\right)^{n}}}\right) \\
=\left(\sum_{n=0}^{\infty} \frac{(1-n) a_{i}}{e^{i n \psi}} \frac{1}{\rho^{n}}\right) \\
\cdot\left(\sum_{n=0}^{\infty} \frac{(1-n) \overline{a_{i}}}{e^{-i n \psi}} \frac{1}{\rho^{n}}\right)
\end{aligned}
$$

Let $a_{i}$ and $b_{i}$ be two infinite series with complex terms. The Cauchy product of these two infinite series is defined by a discrete convolution as follows:

$$
\left(\sum_{i=0}^{\infty} a_{i}\right) \cdot\left(\sum_{j=0}^{\infty} b_{i}\right)=\sum_{k=0}^{\infty} c_{k}
$$

where

$$
c_{k}=\sum_{l=0}^{k} a_{l} b_{k-l}
$$

Then

$$
\left(\sum_{i=0}^{\infty} a_{i} x^{-i}\right) \cdot\left(\sum_{j=0}^{\infty} b_{i} x^{-j}\right)=\sum_{k=0}^{\infty} c_{k} x^{-k}
$$


Proc. of the Seventh International Conference On Advances in Applied Science and Environmental Engineering - ASEE 2017. Copyright (C) Institute of Research Engineers and Doctors. All rights reserved.

ISBN: 978-1-63248-125-2 doi: 10.15224/ 978-1-63248-125-2-10

where

Introducing constant $\lambda$ and let

$$
c_{k}=\sum_{l=0}^{k} a_{l} b_{k-l}
$$

Apply to (17), we have

where

$$
\left|\frac{\partial z}{\partial \zeta}\right|^{2}=\sum_{n=0}^{\infty} c(\psi)_{n} \rho^{-n}
$$

$$
\begin{array}{r}
c(\psi)_{n}=\sum_{j=0}^{n}\left((1-j) a_{j} / e^{i j \psi}\right) \\
\cdot\left((1-n+j) \overline{a_{n-j}} / e^{-i(n-j) \psi}\right)
\end{array}
$$

For mutually conjugate series and their products $c(\psi)_{\mathrm{n}}$, it can be seen from the Cauchy product that:

1) $c(\psi)_{n}$ have the same distribution of the non-zero terms as $\mathrm{a}_{\mathrm{i}}$ and $\overline{\mathrm{a}_{\mathrm{i}}}$;

2) $c(\psi)_{0}=a_{0} \overline{a_{0}}>0$, has nothing to do with $\psi$.

Let $\mathrm{R}_{\mathrm{e}}=\left|\mathrm{a}_{0}\right|=\sqrt{\mathrm{a} \overline{\mathrm{a}_{0}}}$ and call it as equivalent scalar to be used later.

Continue using the method of separation of variables, and let

$$
\varphi=R(\rho) \Theta(\psi)
$$

It turns to be

$$
\Delta(\mathrm{R}(\rho) \Theta(\psi))+\mathrm{k}^{2} \sum_{\mathrm{n}=0}^{\infty} \mathrm{c}(\psi)_{\mathrm{n}} \rho^{-\mathrm{n}} \mathrm{R}(\rho) \Theta(\psi)=0
$$

In the polar system

$$
\begin{aligned}
& \frac{\left(\frac{d}{d \rho} R(\rho)\right) \Theta(\psi)+\rho\left(\frac{d^{2}}{d \rho^{2}} R(\rho)\right) \Theta(\psi)+R(\rho) \frac{\left(\frac{d^{2}}{d \psi^{2}} \Theta(\psi)\right)}{\rho}}{\rho} \\
& +k^{2}\left(\sum_{n=0}^{\infty} c(\psi)_{n} \rho^{-n}\right) R(\rho) \Theta(\psi)=0
\end{aligned}
$$

By dividing $\Theta(\psi)$ and rearranging, we have:

$$
\begin{aligned}
\frac{d^{2}}{d \rho^{2}} R(\rho)+\frac{1}{\rho} \frac{d}{d} \rho R(\rho) & +\frac{R(\rho)}{\rho^{2}}\left(\frac{d^{2}}{d \psi^{2}} \Theta(\psi)\right) \\
& +k^{2}\left(\sum_{n=0}^{\infty} c(\psi)_{n} \rho^{-n}\right) R(\rho)=0
\end{aligned}
$$

\section{B. Simplification and solution of expanded Bessel equation}

For convenience, rewrite equation is of the form

$$
y^{\prime \prime}+P(x) y^{\prime}+Q(x) y=0
$$

With natural boundary condition, it becomes to be an eigenvalue problem

$$
\begin{gathered}
\lambda=m^{2}(m=1,2,3 \ldots) \\
\Theta=A \cos m \psi+B \cos m \psi
\end{gathered}
$$

Substitute into (19), we have

$$
\begin{aligned}
\frac{d^{2}}{d \rho^{2}} R(\rho)+\frac{1}{\rho} \frac{d}{d} \rho R & (\rho) \\
& +\left(\frac{-m^{2}}{\rho^{2}}+k^{2}\left(\sum_{n=0}^{\infty} c(\psi)_{n} \rho^{-n}\right)\right) R(\rho) \\
& =0
\end{aligned}
$$

And with variable substitution $\mathrm{x}=\mathrm{k} \rho$ it becomes to be:

$$
\frac{d^{2}}{d x^{2}} R(x)+\frac{1}{x} \frac{d}{d x} R(x)+\left(\sum_{n=0}^{\infty} c(\psi)_{n} \frac{k^{n}}{x^{n}}-\frac{m^{2}}{x^{2}}\right) R(x)=0
$$

For simplicity, let $\mathrm{b}_{\mathrm{n}}=\mathrm{c}(\psi)_{\mathrm{n}} \mathrm{k}^{\mathrm{n}}$ and we have

$$
\frac{d^{2}}{d x^{2}} R(x)+\frac{1}{x} \frac{d}{d x} R(x)+\left(\sum_{n=0}^{\infty} \frac{b_{n}}{x^{n}}-\frac{m^{2}}{x^{2}}\right) R(x)=0
$$

From (20), it is similar with Bessel equation, but the coefficients of $\mathrm{R}(\mathrm{x})$ contain negative power series of $\mathrm{x}$ and varies with $\psi$, which makes the equation more complex. In can be retrieved to Bessel equation, so it is called as Expanded Bessel Equation in this paper.

where

$$
\begin{gathered}
P(x)=\frac{1}{x} \\
Q(x)=\sum_{n=0}^{\infty} \frac{b_{n}}{x^{n}}-\frac{m^{2}}{x^{2}}=\sum_{n=0}^{\infty} \frac{B_{n}}{x^{n}}
\end{gathered}
$$

with $\mathrm{x}=0$ as its infinite order irregular singularity point. It cannot be solved by Frobenius series directly $[11,12]$. 
In practical application, infinite series $Q(x)$ needs to be truncated. Say truncated to $\mathrm{N}$ terms, for $\mathrm{B}_{\mathrm{n}}, \mathrm{B}_{\mathrm{n}, \mathrm{n} \neq 2}=$ $b_{n}, B_{2}=b_{2}-m^{2}, n=0 . . N$. For convenience, let $\mathrm{N}=2 \mathrm{k}+2,(\mathrm{k} \geq 1)$, then $\mathrm{x}=0$ is its singularity point with rank $\mathrm{k}$.At $\mathrm{x} \rightarrow 0$,its solution can be written as:

$$
y(x)=e^{E(x)} F(x)
$$

where

$$
\begin{array}{r}
E(x)=\sum_{j=1}^{k} \frac{A_{j}}{x^{j}} \\
F(x)=x^{s} \sum_{l=0}^{\infty} a_{n} x^{1}
\end{array}
$$

$\mathrm{F}(\mathrm{x})$ is the Frobenius series, and $\mathrm{E}(\mathrm{x})$ can be determined by Dominate balance method $[11,12]$.

Since ocean wave's periods usually between 3 to 20 seconds, and considering:

1)the wavenumber $\mathrm{k}$ is less than 1 when period greater than 2 seconds, the coefficient $B_{n}$ decays rapidly with $n$ to 0 as it is proportional to $\mathrm{k}^{\mathrm{n}}$;

2)and if truncated more than -2 powers, $A_{j}$ in $E(x)=$ $\sum_{j=1}^{k} \frac{A_{j}}{x^{j}}$ is an imaginary number, which makes its solution oscillate near $\mathrm{x} \rightarrow 0$ in high frequency and towards to 1 away from zero points;

3 ) the mapping function needs to be truncated too, so it is of accuracy to truncated to -2 degrees.

Thus, only $B_{n}$ truncated to $B_{2}\left(B_{2} \neq 0\right)$ is discussed in this paper.

The truncated equation is

$$
\mathrm{y}^{\prime \prime}+\frac{1}{\mathrm{x}} \mathrm{y}^{\prime}+\left(\mathrm{B}_{0}+\frac{\mathrm{B}_{1}}{\mathrm{x}}+\frac{\mathrm{B}_{2}}{\mathrm{x}^{2}}\right) \mathrm{y}=0
$$

Specially, if the cross-section has 3 or more symmetric axes, there will be $b_{2}(\psi)=0$, by the definition $B_{2}=b_{2}-m^{2}$, it is known the equation can be simplified as Bessel equation

$$
y^{\prime \prime}+\frac{1}{x} y^{\prime}+\left(B_{0}-\frac{m^{2}}{x^{2}}\right) y=0
$$

Let $\eta=\sqrt{B_{0}} x=\left|a_{0}\right| x=\left|a_{0}\right| k \rho=R_{e} k \rho$, it can be written as the standard Bessel equation

$$
\mathrm{y}^{\prime \prime}+\frac{1}{\eta} \mathrm{y}^{\prime}+\left(1-\frac{\mathrm{m}^{2}}{\eta^{2}}\right) \mathrm{y}=0
$$

Though we get Bessel equation again in $\zeta$ plane, it is based on following prerequisites:

(1) the incident wavenumber $\mathrm{k}<1$;

(2) The power series in the expanded Bessel equation is truncated with $\mathrm{O}\left(\mathrm{x}^{-3}\right)$;

(3) The cross-section in physical plane has not less than 3 symmetrical axes.

Thus, in mapped plane $\zeta$, the scattered wavenumber changes from $\mathrm{k}$ to $\left|\mathrm{a}_{0}\right| \mathrm{k}$, which means the wave length is scaled $1 / \mathrm{R}_{\mathrm{e}}$. This scale transformation is comparable with the geometry scaling for the cross-section.

For the mapping, which has less than 3 symmetric axes, the equation is not easy to be simplified. Frobenius series expansion can be substituted into it to solve it:

Multiply the equation with $x^{2}$, then by substituting $\mathrm{y}(\mathrm{x})=\mathrm{x}^{\mathrm{s}} \sum_{\mathrm{i}=0}^{\infty} \mathrm{a}_{\mathrm{n}} \mathrm{x}^{\mathrm{i}}$ and rearrange, we get

$$
\begin{gathered}
s^{2} a_{0}+a_{0} B_{2}+\left(s^{2} a_{1}+a_{1} B_{2}+2 s a_{1}+a_{0} B 1+a_{1}\right) x \\
+\sum_{\substack{i=2 \\
+}}\left(s^{2} a_{i}+a_{i-2} B_{0}+a_{i-1} B_{1}+a_{i} B_{2}\right. \\
\left.+2 i a_{i}+i^{2} a_{i}\right) x^{i}=0
\end{gathered}
$$

By letting the coefficients of $\mathrm{x}$ power equal zero, for the constant term, we have

$$
\mathrm{s}^{2} \mathrm{a}_{0}+\mathrm{a}_{0} \mathrm{~B}_{2}=0
$$

This is the determining-equation of the equation, and

$$
\mathrm{s}= \pm \sqrt{-\mathrm{B}_{2}}
$$

For x's coefficient we have

$$
a_{1}=-\frac{a_{0} B_{1}}{(s+1)^{2}+B_{2}}
$$

And for $x^{i}(i>1)$

$$
a_{i}=-\frac{a_{i-2} B_{0}+a_{i-1} B_{1}}{(s+i)^{2}+B_{2}}
$$

For given $m$,say $s_{1}=\sqrt{-B_{2}}, s_{2}=-\sqrt{-B_{2}}$

One of its solution can be written as

$$
y_{1}(x)=j_{m}(x)=x^{s_{1}} \sum_{i=0}^{\infty} a_{i} x^{i}
$$

Which can be called as its m order solution. 
Proc. of the Seventh International Conference On Advances in Applied Science and Environmental Engineering - ASEE 2017. Copyright (C) Institute of Research Engineers and Doctors. All rights reserved.

ISBN: 978-1-63248-125-2 doi: 10.15224/ 978-1-63248-125-2-10

$$
\mathrm{j}_{-\mathrm{m}}(\mathrm{x})=\mathrm{x}^{\mathrm{s}_{2}} \sum_{\mathrm{i}=0}^{\infty} \mathrm{a}_{\mathrm{i}} \mathrm{x}^{\mathrm{i}}
$$

First, considering the case with $-\mathrm{B}_{2} \geq 0$, or $\mathrm{s}$ is real number, another linear independence solution can be written as

$$
y_{2}(x)=y_{m}(x)=\frac{j_{m}(x) \cos (s \pi)-j_{-m}(x)}{\sin (s \pi)}
$$

Similarly, for $-\mathrm{B}_{2} \leq 0, \mathrm{~s}$ is imaginary number, another linear independence solution can be written as

$$
y_{2}(x)=y_{m}(x)=\frac{j_{m}(x) \Re e^{s \pi}-j_{-m}(x)}{\mathfrak{J} e^{s \pi}}
$$

Where $\mathfrak{R}, \mathfrak{J}$ are Real and Image symbols.

\section{Scattering problem of Multi- symmetrical axes cross-section vertical cylinder}

As discussed before, for the cross-section with 3 and more symmetrical axes, it's scatter wave potential follows Bessel equation.

By referring the scattered wave potential for circular cylinder previous, the scattered wave potential in mapped plane $\zeta$ can be assumed in the similar form of:

$$
\Phi_{\mathrm{S}}=\sum_{\mathrm{m}=0}^{\infty} \mathrm{A}_{\mathrm{m}}(\psi) \mathrm{H}_{\mathrm{m}}^{1}\left(\mathrm{R}_{\mathrm{e}} \mathrm{k} \rho\right) \cos (\mathrm{m}(\psi-\beta))
$$

Where $A_{m}(\psi)$ is the amplitude of m order for $\psi$.

We'll go through in solving $A_{m}(\psi)$ below:

In mapped plane $\zeta$, the cross-section boundary is mapped onto $\rho=1$ circle. The boundary condition for scattering problem is:

$$
\frac{\partial \Phi_{\mathrm{I}}}{\partial \mathrm{n}}=-\frac{\partial \Phi_{\mathrm{S}}}{\partial \mathrm{n}}
$$

And with the assumption that every order of both incident and scattered wave potential satisfies the boundary condition correspondently.

For each order, the following equation holds:

$$
\begin{aligned}
\frac{\partial \Phi_{\mathrm{Sm}}}{\partial \mathrm{n}}=\frac{\partial \Phi_{\mathrm{Sm}}}{\partial \rho}= & \mathrm{A}_{\mathrm{m}}(\psi) \mathrm{R}_{\mathrm{e}} \mathrm{kH}_{\mathrm{m}}^{1}{ }^{\prime}\left(\mathrm{R}_{\mathrm{e}} \mathrm{k} \rho\right) \cos (\mathrm{m}(\psi \\
& -\beta)),(\rho=1)
\end{aligned}
$$

And for its physical meaning as velocity, it can be known that $\frac{\partial \Phi_{I m}}{\partial \mathrm{n}}$ 's direction is in the same line as $\rho$ and vertical to $\psi$ 's direction.

By applying full derivative rule, we have

$$
\frac{\partial \Phi_{\operatorname{Im}}}{\partial \rho}=\frac{\partial \Phi_{\operatorname{Im}}}{\partial \mathrm{r}} \frac{\partial \mathrm{r}}{\partial \rho}+\frac{\partial \Phi_{\operatorname{Im}}}{\partial \theta} \frac{\partial \theta}{\partial \rho}
$$

In which

$$
\frac{\partial \Phi_{\mathrm{Im}}}{\partial \mathrm{r}}=\mathrm{k}_{\mathrm{m}} \mathrm{i}^{\mathrm{m}} \mathrm{J}_{\mathrm{m}}{ }^{\prime}(\mathrm{kr}) \cos (\mathrm{m}(\theta-\beta))
$$

$$
\frac{\partial \Phi_{\mathrm{Im}}}{\partial \theta}=-\mathrm{m} \epsilon_{\mathrm{m}} \mathrm{i}^{\mathrm{m}} \mathrm{J}_{\mathrm{m}}(\mathrm{kr}) \sin (\mathrm{m}(\theta-\beta))
$$

where

$$
\zeta_{1}=e^{(i \psi)}, z_{1}=\sum_{n=0}^{\infty} a_{n} \zeta_{1}^{1-n}, r=\left|z_{1}\right|, \theta=\arg \left(z_{1}\right)
$$

And combine with the mapping relation formula obtained previously, we have

$$
\begin{array}{r}
\frac{\partial \Phi_{\mathrm{Im}}}{\partial \rho}=\epsilon_{\mathrm{m}} \mathrm{i}^{\mathrm{m}}\left(\mathrm { e } ^ { \mathrm { i } ( \Psi - \theta ) } \frac { \mathrm { dz } } { \mathrm { d } \zeta } \left[\mathrm{kJ}_{\mathrm{m}}{ }^{\prime}(\mathrm{kr}) \cos (\mathrm{m}(\theta-\beta))\right.\right. \\
\left.\left.+\mathrm{miJ}_{\mathrm{m}}(\mathrm{kr}) \sin (\mathrm{m}(\theta-\beta)) / \mathrm{r}\right]\right)
\end{array}
$$

Let

$$
\begin{aligned}
& \mathrm{u}_{\rho}=\mathrm{e}^{\mathrm{i}(\psi-\theta)} \frac{\mathrm{dz}}{\mathrm{d} \zeta}\left[\mathrm{kJ}_{\mathrm{m}}{ }^{\prime}(\mathrm{kr}) \cos (\mathrm{m}(\theta-\beta))\right. \\
& \left.+\operatorname{miJ}_{\mathrm{m}}(\mathrm{kr}) \sin (\mathrm{m}(\theta-\beta)) / \mathrm{r}\right]
\end{aligned}
$$

$\mathrm{u}_{\rho}$ is a complex number, which means it's a velocity vector with $\rho$ as its baseline in the plane, its real part shares the same line with $\rho$ in direction, while its imaginary part is vertical to $\rho$ and in the same direction as $\psi$. Then, we can say

$$
\frac{\partial \Phi_{\mathrm{Im}}}{\partial \mathrm{n}}=\epsilon_{\mathrm{m}} \mathrm{i}^{\mathrm{m}} \mathrm{u}_{\mathrm{n}}=\epsilon_{\mathrm{m}} \mathrm{i}^{\mathrm{m}} \mathfrak{R}\left(\mathrm{u}_{\rho}\right)
$$

Similarly, if take $\psi$ as the vector baseline, we also have 
Proc. of the Seventh International Conference On Advances in Applied Science and Environmental Engineering - ASEE 2017. Copyright (C) Institute of Research Engineers and Doctors. All rights reserved.

ISBN: 978-1-63248-125-2 doi: 10.15224/ 978-1-63248-125-2-10

$$
\begin{array}{r}
\frac{\partial \Phi_{\mathrm{Im}}}{\partial \psi}=\epsilon_{\mathrm{m}} \mathrm{i}^{\mathrm{m}}\left(\mathrm { e } ^ { \mathrm { i } ( \Psi - \theta ) } \frac { \mathrm { dz } } { \mathrm { d } \zeta } \left[\mathrm{i} \rho \mathrm{kJ} \mathrm{m}_{\mathrm{m}}{ }^{\prime}(\mathrm{kr}) \cos (\mathrm{m}(\theta-\beta))\right.\right. \\
\left.\left.-\mathrm{m} \rho \mathrm{J}_{\mathrm{m}}(\mathrm{kr}) \sin (\mathrm{m}(\theta-\beta)) / \mathrm{r}\right]\right)
\end{array}
$$

Let,

$$
\begin{aligned}
& \mathrm{u}_{\Psi}=\mathrm{e}^{\mathrm{i}(\psi-\theta)} \frac{\mathrm{dz}}{\mathrm{d} \zeta}\left[\mathrm{i} \rho \mathrm{kJ} \mathrm{m}^{\prime}{ }^{\prime}(\mathrm{kr}) \cos (\mathrm{m}(\theta-\beta))\right. \\
& \left.-\mathrm{m} \rho \mathrm{J}_{\mathrm{m}}(\mathrm{kr}) \sin (\mathrm{m}(\theta-\beta)) / \mathrm{r}\right]
\end{aligned}
$$

Then we know it's a velocity vector with $\psi$ as its baseline, its real part shares the same line with $\psi$ in direction, while its imaginary part is $\pi / 2$ plus $\psi$ 's direction.

On the boundary of unit circle, also holds:

$$
\begin{aligned}
\mathrm{u}_{\mathrm{n}}= & \Re\left(\mathrm{u}_{\psi} / \mathrm{i}\right)=\mathfrak{R}\left(\mathrm { e } ^ { \mathrm { i } ( \psi - \theta ) } \frac { \mathrm { dz } } { \mathrm { d } \zeta } \left[\rho \mathrm{kJ} \mathrm{J}_{\mathrm{m}}^{\prime}(\mathrm{kr}) \cos (\mathrm{m}(\theta-\beta))+\right.\right. \\
& \left.\left.\operatorname{im} \rho \mathrm{J}_{\mathrm{m}}(\mathrm{kr}) \sin (\mathrm{m}(\theta-\beta)) / \mathrm{r}\right]\right)=\mathfrak{R}\left(\mathrm{u}_{\rho}\right),(\rho=1)
\end{aligned}
$$

The treatment above-mentioned is the key to handle boundary condition along mapped unit circle, it is a general way for such mapped boundary.

Then we have:

$$
\mathrm{A}_{\mathrm{m}}(\psi)=-\left(\epsilon_{\mathrm{m}} \mathrm{i}^{\mathrm{m}} \mathfrak{R}\left(\mathrm{u}_{\rho}\right)\right) /\left(\mathrm{kRH}_{1}{ }^{\prime}\left(\mathrm{kR}_{\mathrm{e}}\right) \cos (\mathrm{m}(\psi-\beta))\right)
$$

,$(\rho=1)$

Till now, we have finished the potential solution for bottom -mounted vertical cylinder with non-circular cross-section.

Then we have the diffraction potential as

$$
\Phi_{\mathrm{D}}=\Phi_{\mathrm{I}}+\Phi_{\mathrm{S}}
$$

For any point $(\rho, \psi)$ in plane $\zeta$,we can get the potential at corresponding point $(r, \theta)$ by using the mapping function, then we have $\Phi_{\mathrm{I}}$ at that point. Similar, we can get $\Phi_{S}, \Phi_{D}$ in $\mathrm{z}$ plane the same way.

Usually, the following physical quantities are of interesting in engineering and can be obtained from wave potential:

Wave profile $\eta$

$$
\Phi=-\frac{\text { ig }}{\omega} \mathrm{f}_{0}(\mathrm{z}) \eta
$$

Dynamic pressure $\mathrm{p}$

$$
\mathrm{p}=\mathrm{i} \omega \rho_{\mathrm{s}} \Phi
$$

where $\rho_{\mathrm{s}}$ is the sea-water density.

\section{Iv. Validation cases}

A C++ program CTCWS (Conformal Transformation based Cylinder Wave Solver) is development based on the methods of this paper, which calculations section mapping function, wave potential, wave elevation, pressure and wave load. With the program, special designed cases are investigated for this paper.

The validation objective of the cases is : Compare wave potential related results with BEM to valid the theory assumption proposed in this paper, to see if it is suitable for engineering.

\section{A. Case 1: square cross section vertical cylinder}

Input parameters:

A bottom-mounted vertical cylinder, with:

$\checkmark \quad$ Water depth:100m;

$\checkmark$ Cross-section: square, with side length $16 \mathrm{~m}$;

$\checkmark \quad$ Gravity acc.: $\mathrm{g}=9.81 \mathrm{~m} / \mathrm{s}^{2}$

$\checkmark \quad$ Density of seawater: $\rho_{\mathrm{s}}=1025.0 \mathrm{~kg} / \mathrm{m}^{3}$

Results calculated:

(1) a plane wave with $\mathrm{T}=6 \mathrm{~s}$, heading $0^{\circ}, 45^{\circ}$, to investigate the similarities and differences of wave potential between method of this paper and BEM.

(2) wave loads with $\mathrm{T}=2,3,4,5,6,7,8,9,10,11,12,13,14,15 \mathrm{~s}$, and heading $0^{\circ}, 15^{\circ}, 30^{\circ}, 45^{\circ}$. To investigate reliability of wave loads of the proposed method.

\section{Results:}

(1) The coefficients of mapping function of the cross-section to unit circle.

TABLE I.

COEFFICIENTS OF SQUARE

\begin{tabular}{|c|c|c|}
\hline $\begin{array}{c}\text { Non-zero term } \\
\text { index }\end{array}$ & Real part & $\begin{array}{c}\text { Imaginary } \\
\text { part }\end{array}$ \\
\hline 0 & 9.446507 & 0 \\
\hline 4 & -1.579451 & 0 \\
\hline 8 & 0.171893 & 0 \\
\hline 12 & -0.056166 & 0 \\
\hline 16 & 0.026634 & 0 \\
\hline 20 & -0.015226 & 0 \\
\hline 24 & 0.009575 & 0 \\
\hline 28 & -0.006011 & 0 \\
\hline 32 & 0.002245 & 0 \\
\hline
\end{tabular}

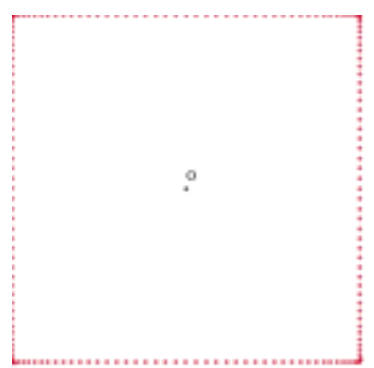

Figure 1. Conformal mapped boundary on unit circle 
Proc. of the Seventh International Conference On Advances in Applied Science and Environmental Engineering - ASEE 2017. Copyright ( Institute of Research Engineers and Doctors. All rights reserved.

ISBN: 978-1-63248-125-2 doi: 10.15224/ 978-1-63248-125-2-10

The validation square with calculated mapping functions applied onto unit circle is shown in figure 1.

The wavenumber is $\mathrm{k}=0.1117862$, omit time term, the incident wave can be written as:

$\eta_{I}=\operatorname{Acos}(\operatorname{krcos}(\theta-\beta)),(r, \theta)$ is the points in polar coordinate. All the calculation is assumed that $\mathrm{A}=1$.

(2) Wave elevation

\section{wave elevation for $\beta=0^{\circ}$}

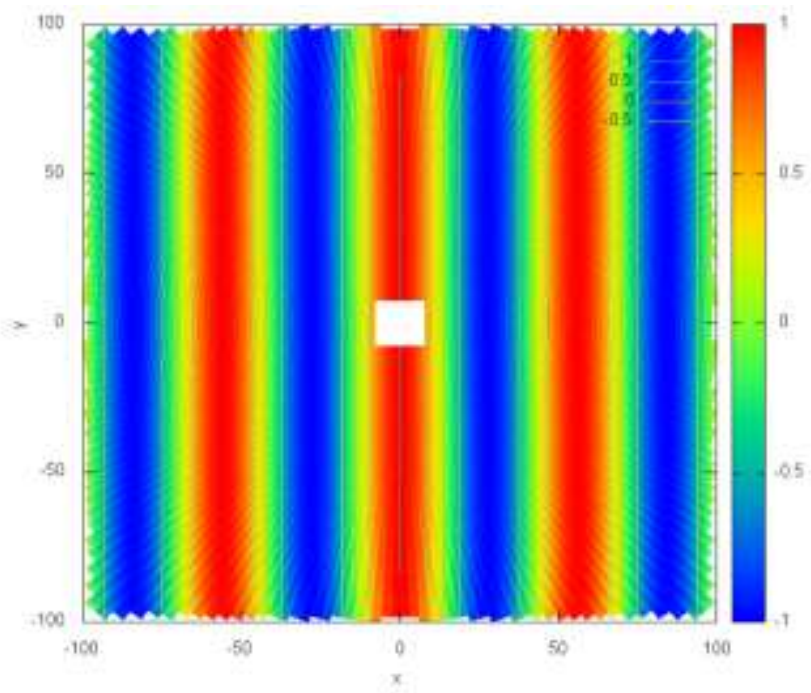

Figure 2. Incident wave elevation in physical plane

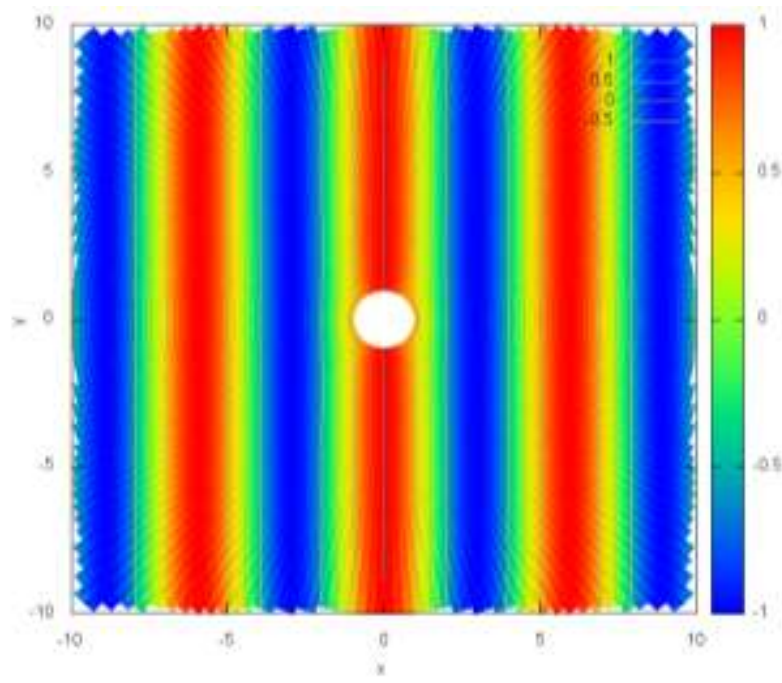

Figure 3. Incident wave elevation in mapped plane

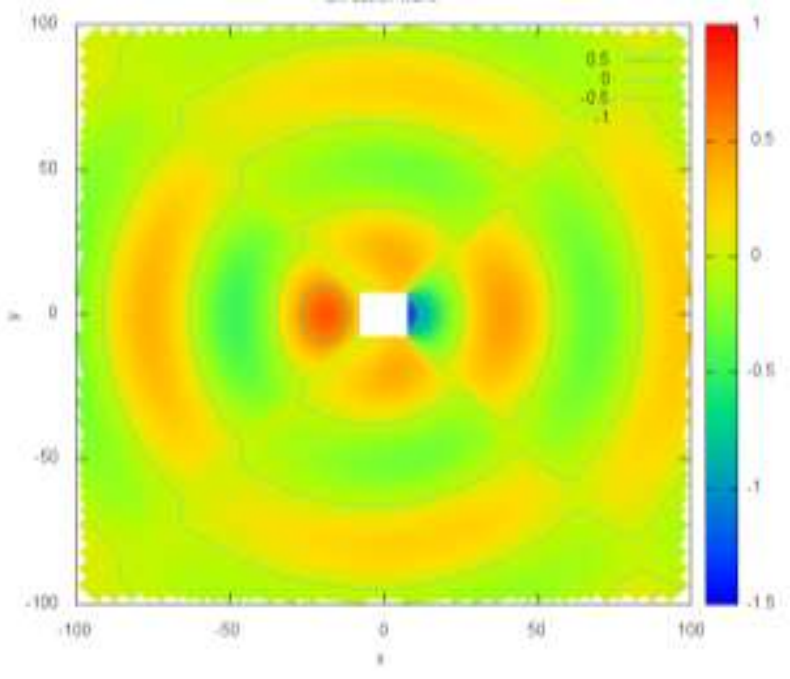

Figure 4. Scattered wave elevation by proposed method in physical plane

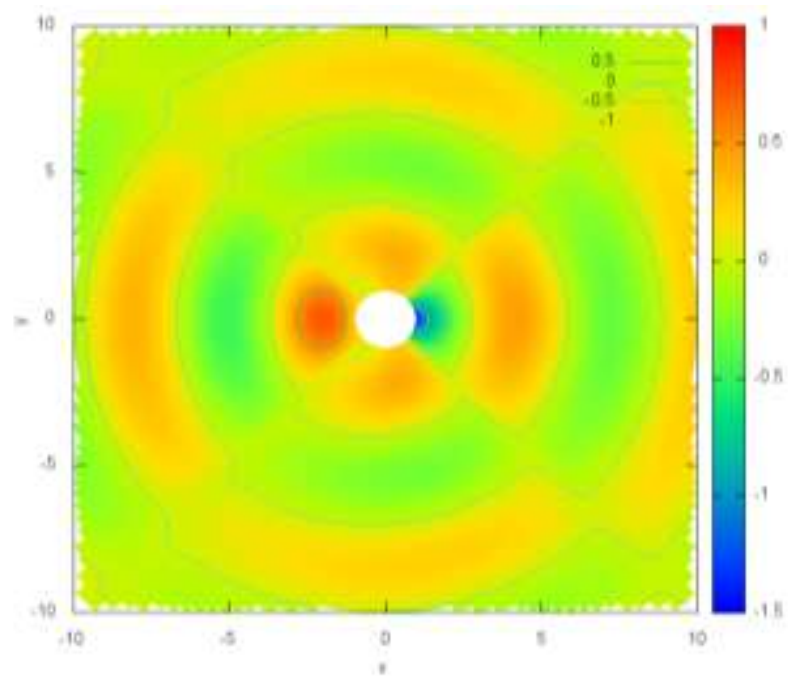

Figure 5. Scattered wave elevation by proposed method in mapped plane

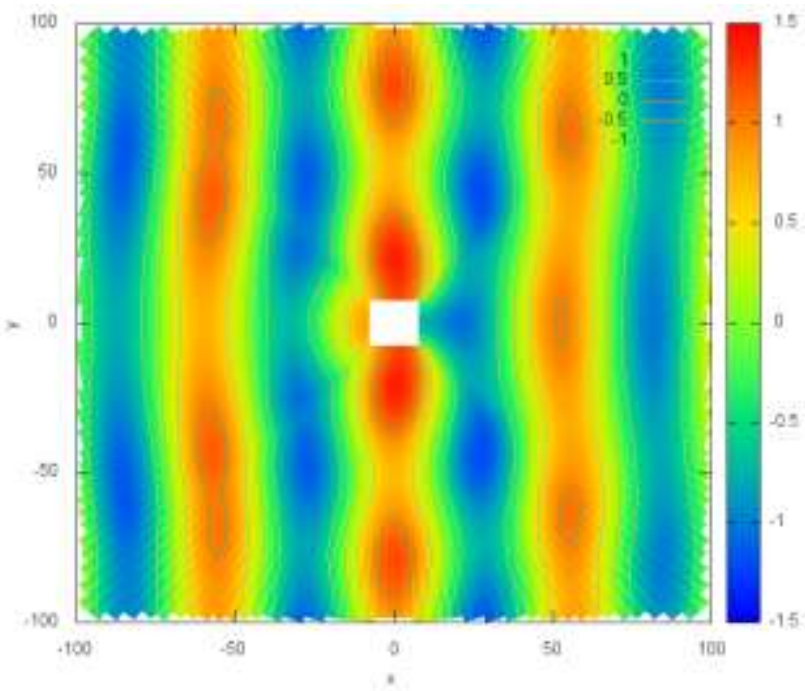

Figure 6. Diffraction wave elevation by proposed method in physical plane 
Proc. of the Seventh International Conference On Advances in Applied Science and Environmental Engineering - ASEE 2017. Copyright $(\odot$ Institute of Research Engineers and Doctors. All rights reserved.

ISBN: 978-1-63248-125-2 doi: 10.15224/ 978-1-63248-125-2-10

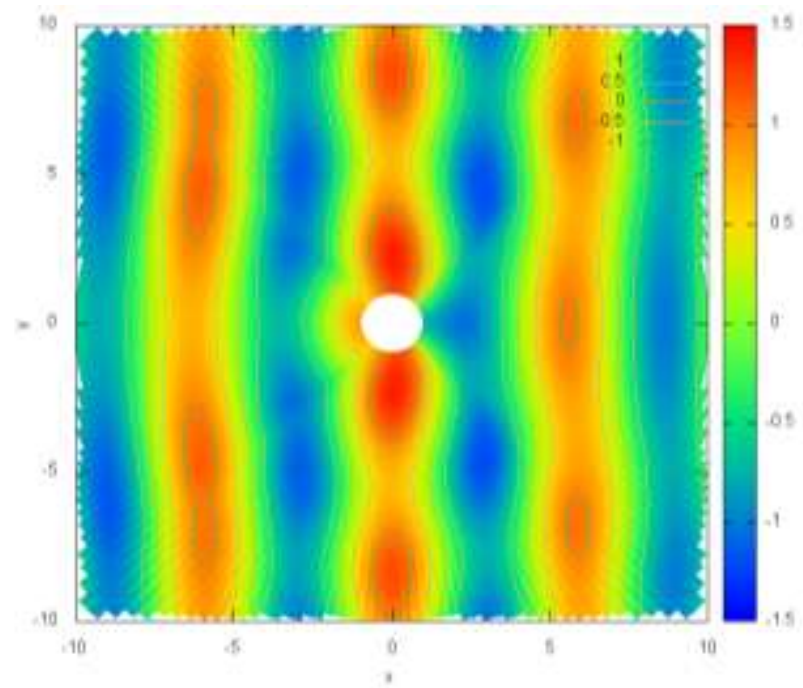

Figure 7. Diffraction wave elevation by proposed method in mapped plane

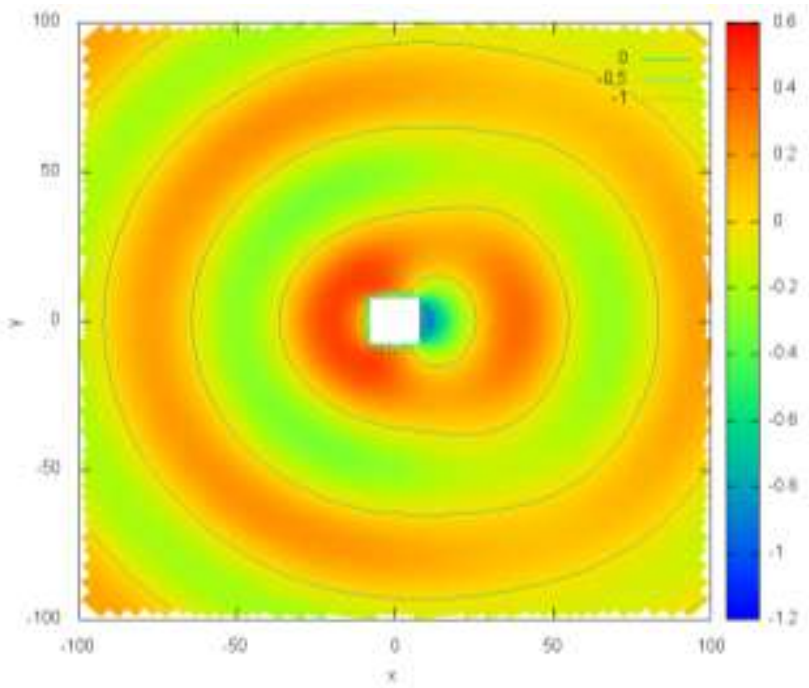

Figure 8. scattered wave elevation by BEM(WAMIT) in physical plane

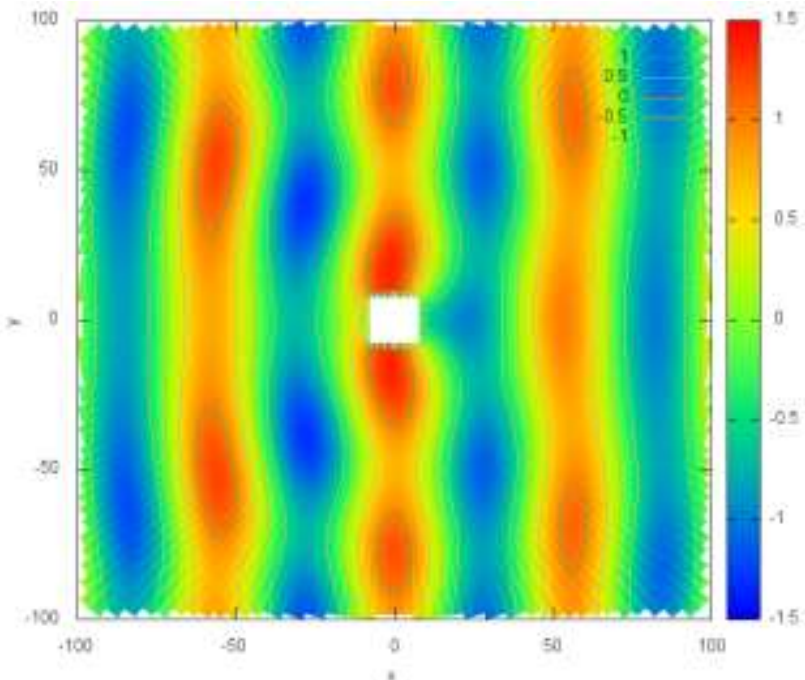

Figure 9. diffraction wave elevation by BEM(WAMIT) in physical plane

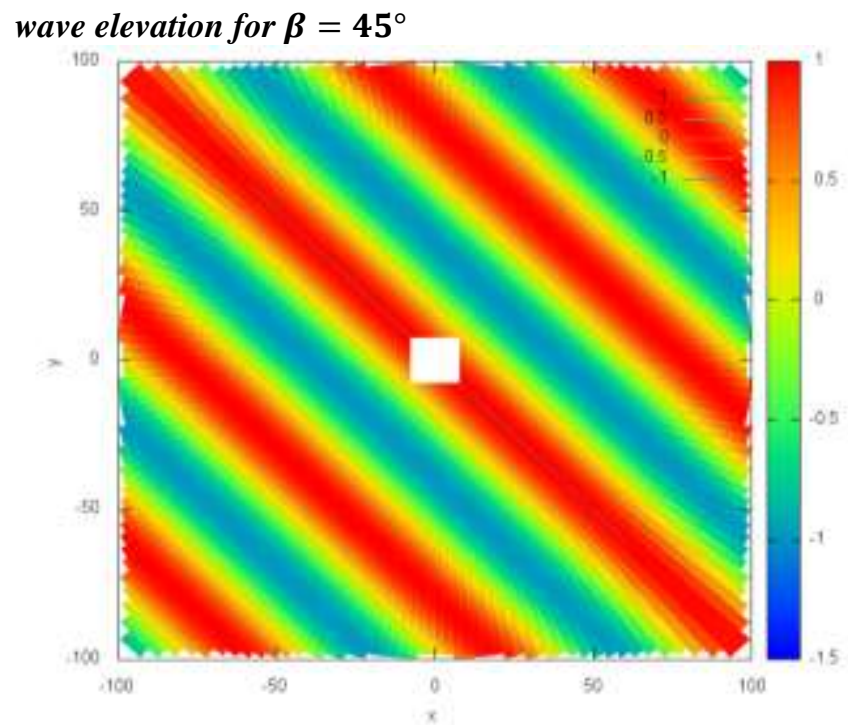

Figure 10. Incident wave elevation in physical plane

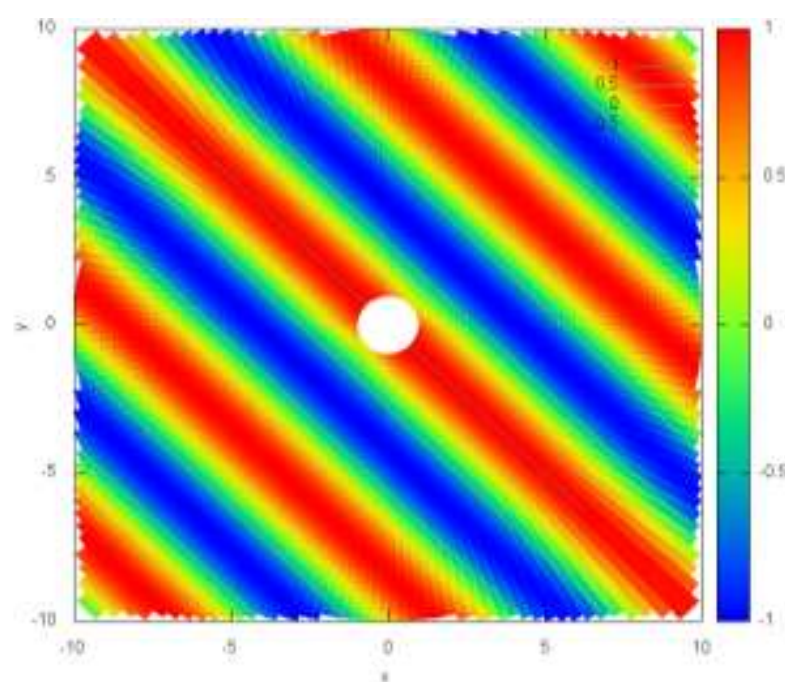

Figure 11. Incident wave elevation in mapped plane

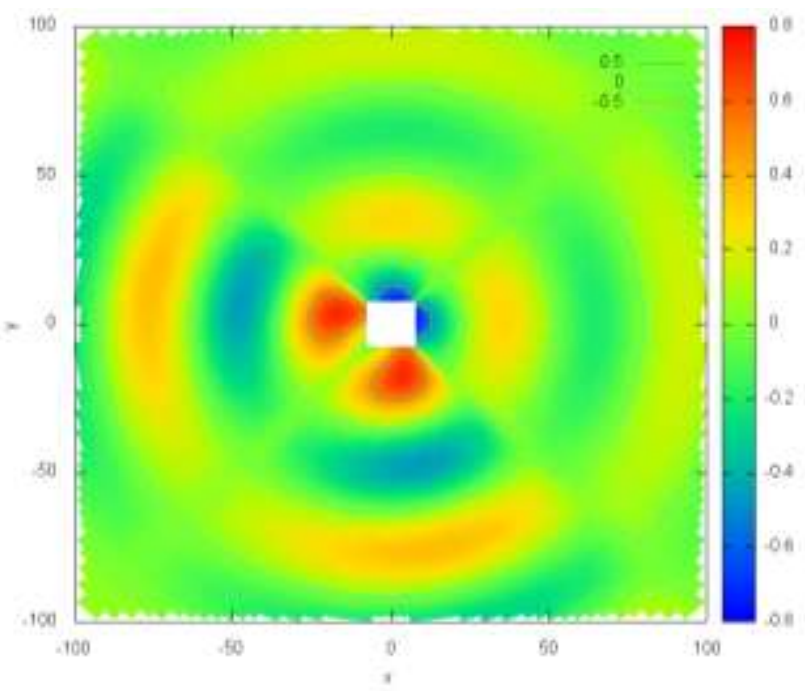

Figure 12. Scattered wave elevation by proposed method in physical plane 
Proc. of the Seventh International Conference On Advances in Applied Science and Environmental Engineering - ASEE 2017. Copyright $\odot$ Institute of Research Engineers and Doctors. All rights reserved.

ISBN: 978-1-63248-125-2 doi: 10.15224/ 978-1-63248-125-2-10

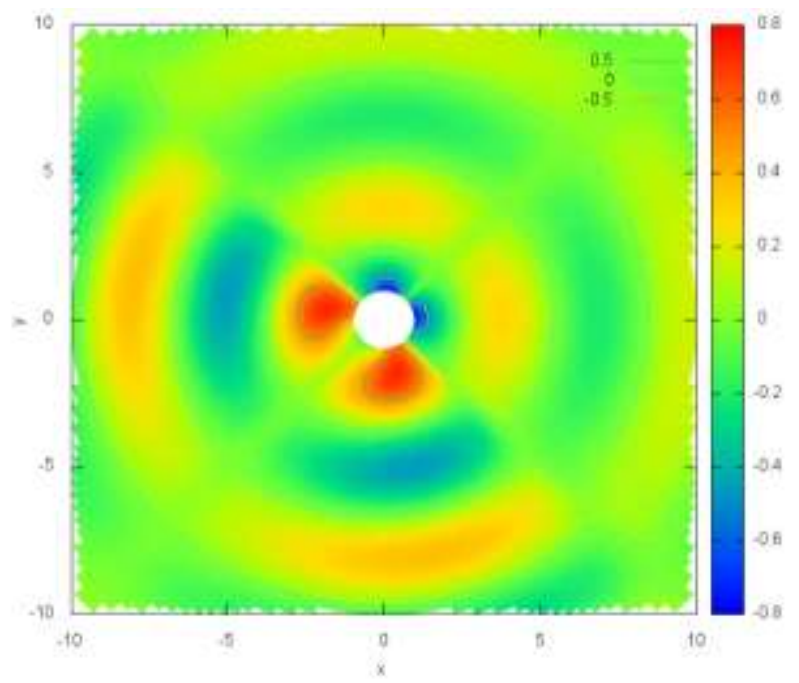

Figure 13. Scattered wave elevation by proposed method in mapped plane

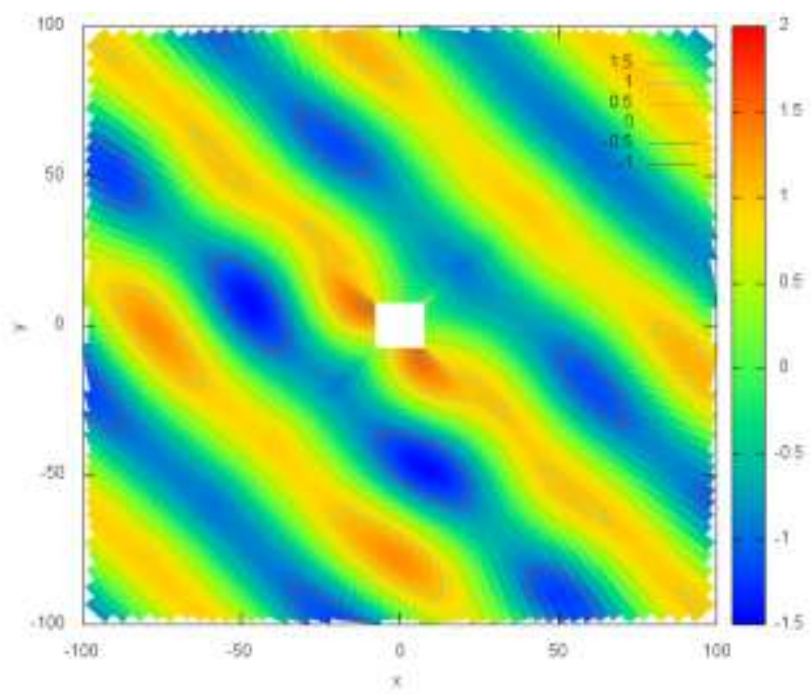

Figure 14. Diffraction wave elevation by proposed method in physical plane

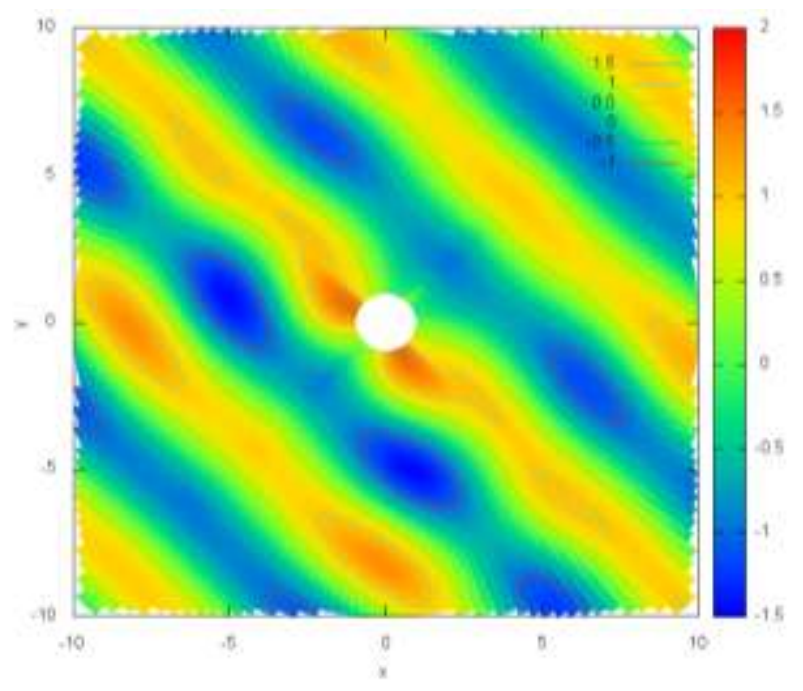

Figure 15. Diffraction wave elevation by proposed method in mapped plane

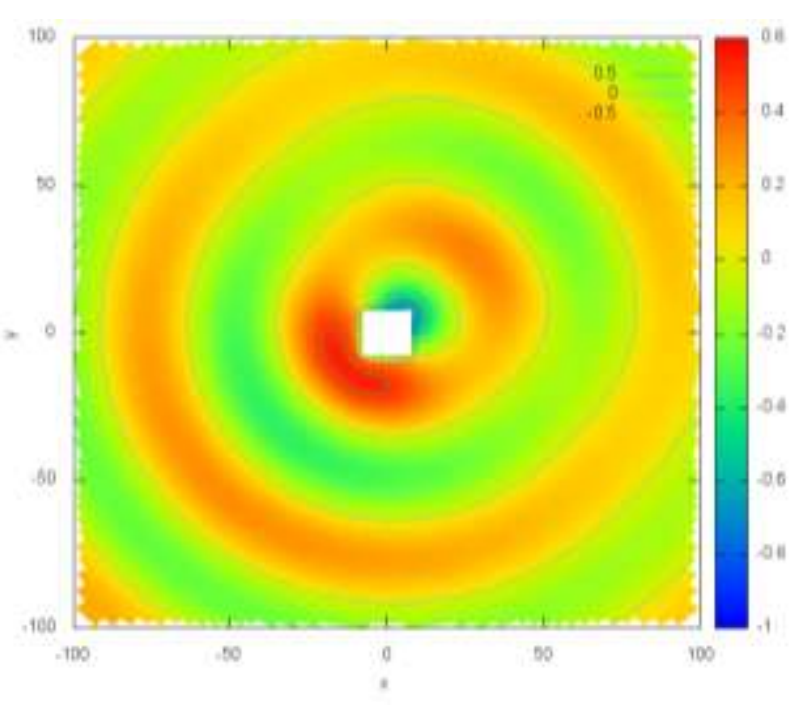

Figure 16. Scattered wave elevation by BEM(WAMIT) in physical plane

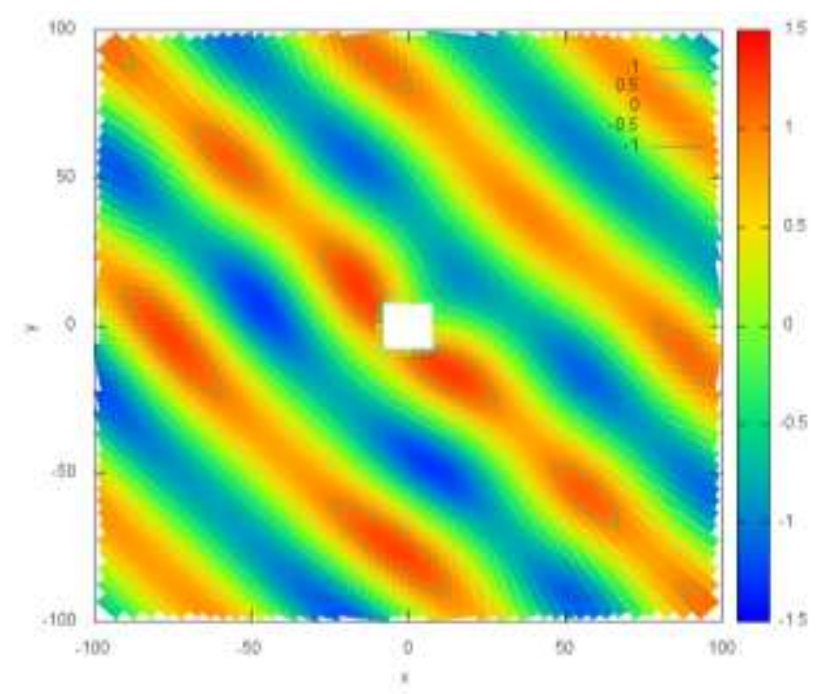

Figure 17. diffraction wave elevation by BEM(WAMIT) in physical plane

From the wave elevation results, it can be concluded:

$\checkmark \quad$ The wave elevation of the proposed method is basically the same as BEM results.

$\checkmark \quad$ the proposed method shows the affection of sharp corner of square section, while BEM method does not. The reason is the that by the proposed method the scatted wave elevation is only determined by the mapping function of cross-section, its position in mapped plane and incident wave parameter, not influenced by points of its neighborhood around circular boundary. While, the results of BEM are by summation of potential on the total wetted surface, which smooths the results.

(3) Wave loads

The wave loads calculated by the method of this paper and BEM(WAMIT) are shown below, where the wave loads are in non-dimensional form $\overline{\mathrm{F}}=\frac{\mathrm{F}}{\rho \mathrm{g}}$ 
Proc. of the Seventh International Conference On Advances in Applied Science and Environmental Engineering - ASEE 2017. Copyright (C) Institute of Research Engineers and Doctors. All rights reserved.

ISBN: 978-1-63248-125-2 doi: 10.15224/ 978-1-63248-125-2-10

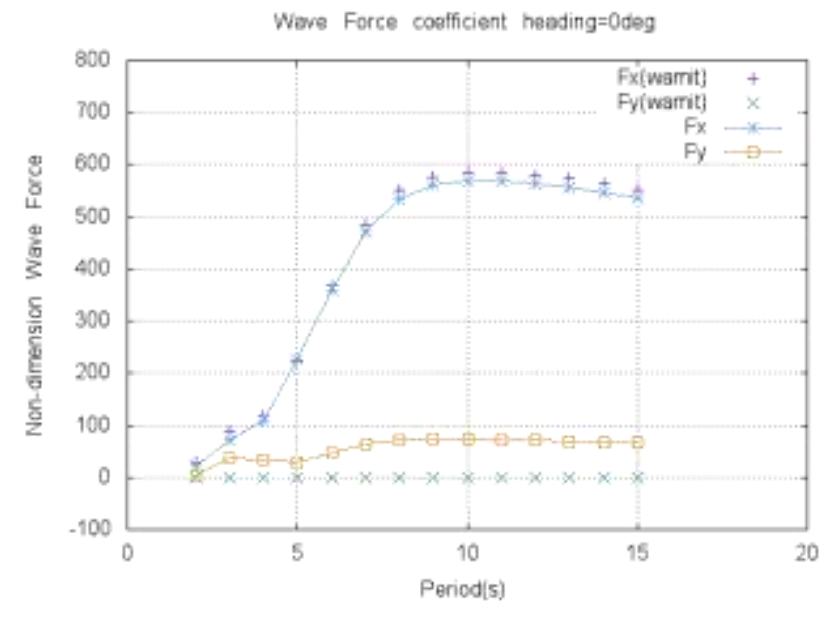

Figure 18. Wave loads amplitude RAO (heading $=0 \mathrm{deg}$ )

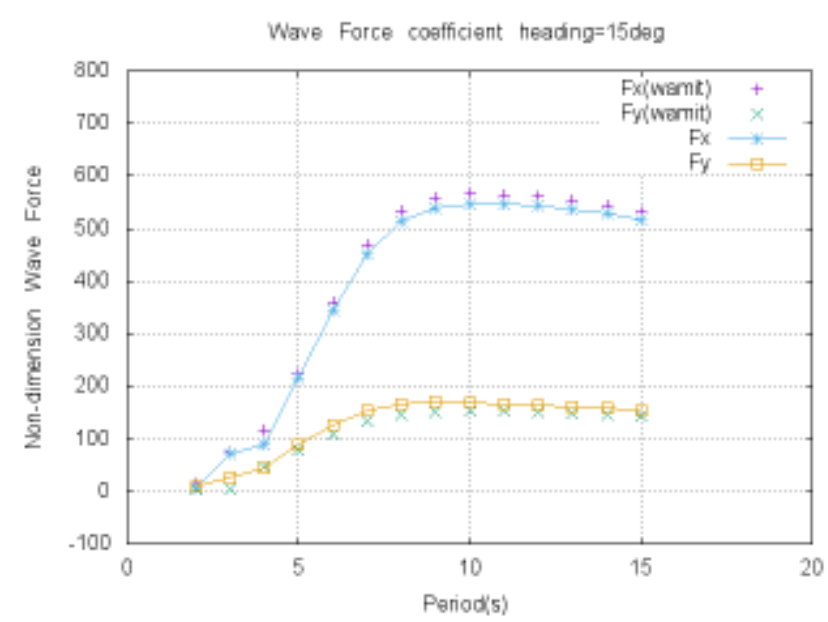

Figure 19. Wave loads amplitude RAO (heading $=15 \mathrm{deg}$ )

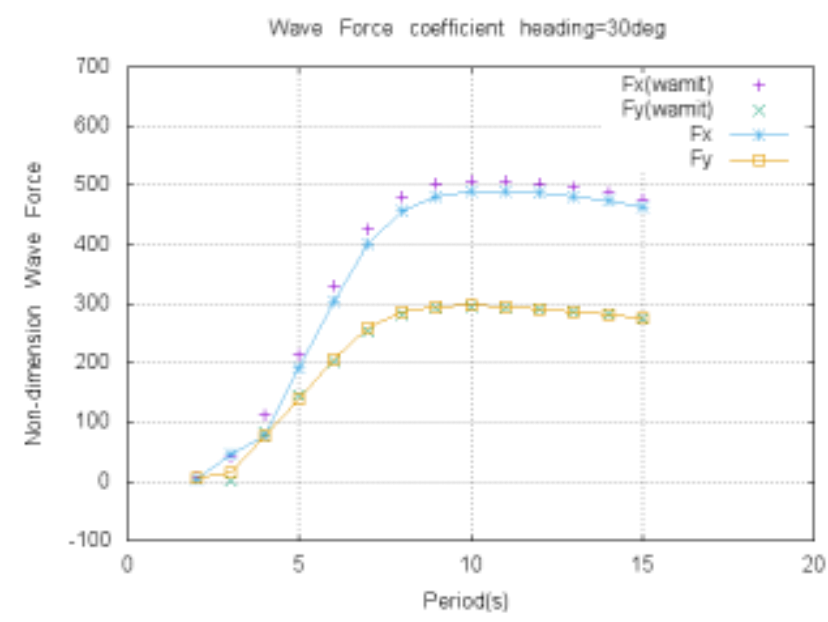

Figure 20. Wave loads amplitude RAO (heading =30deg)

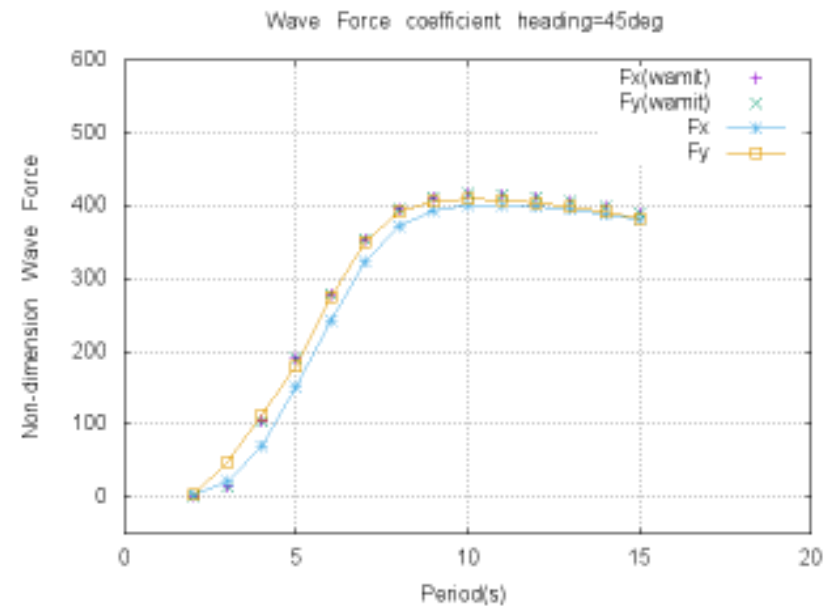

Figure 21. Wave loads amplitude RAO (heading $=45 \mathrm{deg}$ )

The results by the method of this paper is comparable with BEM results, which means the assumption of the method in this paper is reasonable and feasible for engineering.

\section{B. Case2: hexagon cross section vertical cylinder}

To validate the proposed method further, a regular hexagon with $16 \mathrm{~m}$ circumscribed circle diameter is calculated with the same input wave data. The results are shown below.

(1) The mapping function coefficients:

TABLE II. COEFFICIENTS OF HEXAGON

\begin{tabular}{|c|c|c|}
\hline $\begin{array}{c}\text { Non-zero } \\
\text { term index }\end{array}$ & Real part & $\begin{array}{c}\text { Imaginary } \\
\text { part }\end{array}$ \\
\hline 0 & 7.366074 & 0 \\
\hline 6 & -0.496046 & 0 \\
\hline 12 & 0.078193 & 0 \\
\hline 18 & -0.030015 & 0 \\
\hline 24 & 0.016033 & 0 \\
\hline 30 & -0.009837 & 0 \\
\hline 36 & 0.003801 & 0 \\
\hline
\end{tabular}

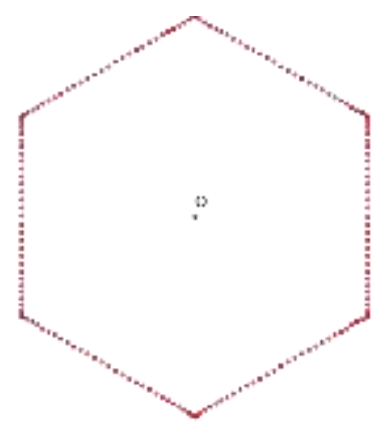

Figure 22. Conformal mapped boundary on unit circle

The validation hexagon with calculated mapping functions applied onto unit circle is shown in figure 22 . 
Proc. of the Seventh International Conference On Advances in Applied Science and Environmental Engineering - ASEE 2017. Copyright (C) Institute of Research Engineers and Doctors. All rights reserved.

ISBN: 978-1-63248-125-2 doi: 10.15224/ 978-1-63248-125-2-10

(2) Wave loads

The wave loads calculated by the method of this paper and BEM(WAMIT) are shown below, where the wave loads are in non-dimensional form $\bar{F}=\frac{F}{\rho g}$

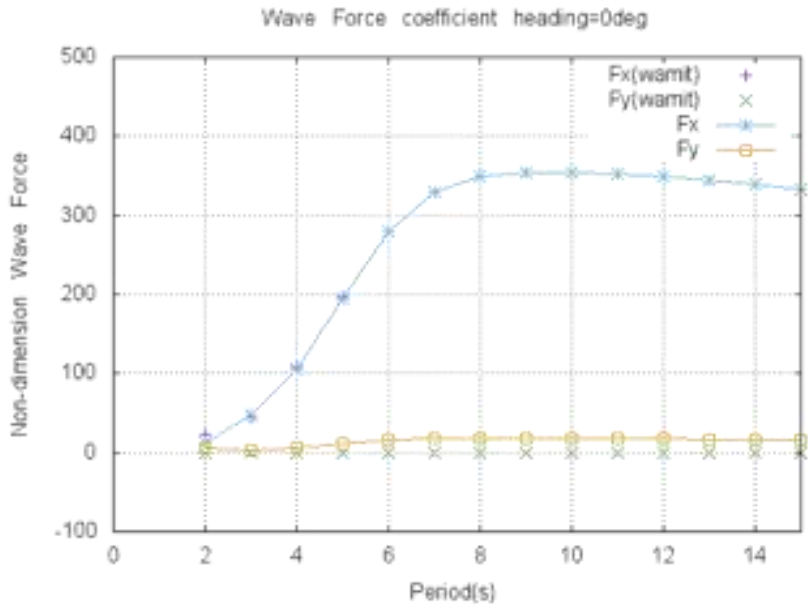

Figure 23. Wave loads amplitude RAO (heading $=0 \mathrm{deg}$ )

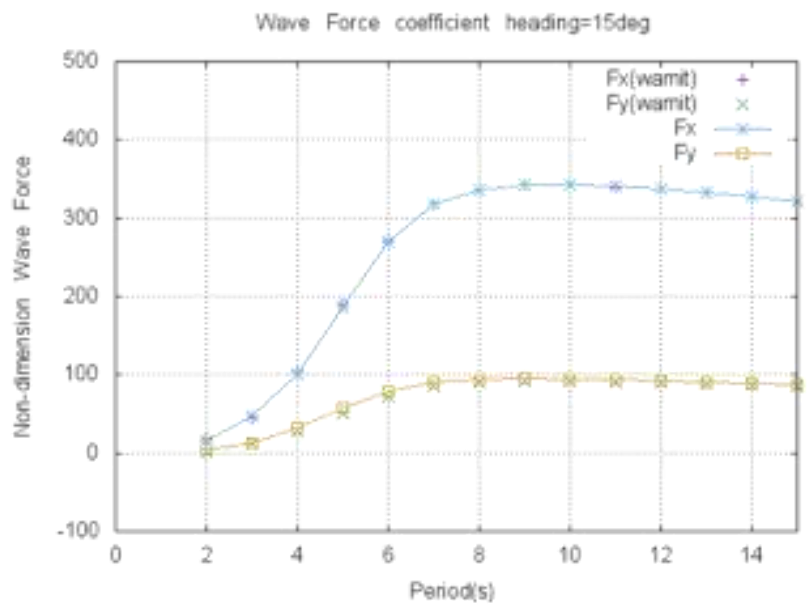

Figure 24. Wave loads amplitude RAO (heading $=15 \mathrm{deg}$ )

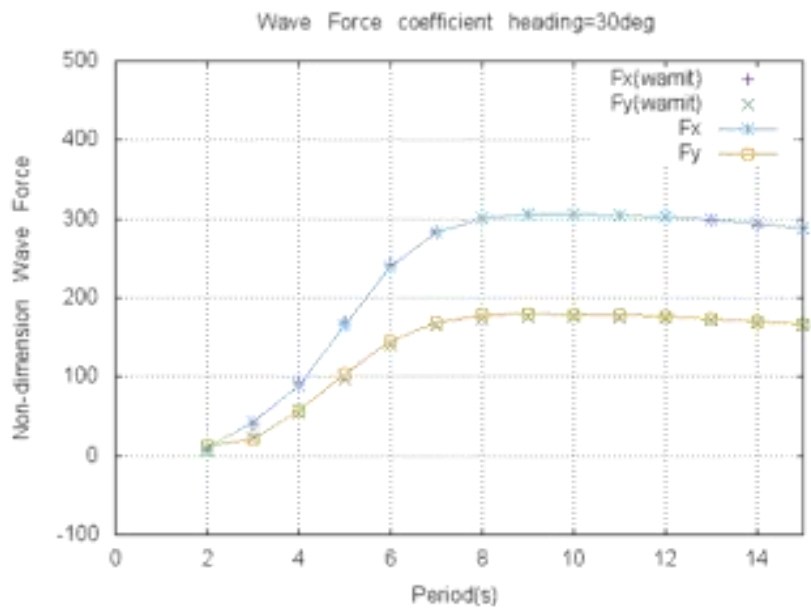

Figure 25. Wave loads amplitude RAO (heading =30deg)

Results of hexagon agree very well with each other, which confirms the feasibility of the method in engineering application.

\section{v.Summary}

In this paper, by referencing the wave scattering of circular cylinder mathematical techniques, combined with conformal transformation in power series to map noncircular cross-section onto unit circle and derived the distorted Helmholtz equation in the mapped plane. With the Expanded Bessel Equation derived, the solutions are also discussed and applied onto the cases with symmetrical axis to get the analytical results of wave scattering problem of non-circular cross-section vertical cylinder. Designed verification cases are calculated to compare the results by the proposed method and BEM, which shows good agreement with each other. It is conformed the method by this paper is suitable for wave loads analysis as a nondiscrete method for non-circular cross-section vertical cylinder.

The model of scattering by a non-circular cross-section is a general basic mathematical problem, which is analyzed with the help of conformal transformation in this paper. The techniques to handle the governing equation and boundary conditions in mapped plane provide reference for the similar problems in other models such as truncated vertical cylinder in hydrodynamics, scattering by cylinder in Acoustic, Electromagnetics etc.

\section{References}

[1] CC Mei, M Stiassnie, D K-P Yue, Theory and Applications of Ocean Surface Waves, World Scientific,2005

[2] C.M. Linton, P. McIver,Handbook of Mathematical Techniques for Wave/Structure Interactions 1st Edition ,Chapman and Hall/CRC,2001

[3] J.M.J. Journée,L.J.M. Adegeest, Theoretical Manual of Strip Theory Program "SEAWAY for Windows" , DUT-SHL Report 1370,September 2003,Sep.

[4] G. R. Mogridge and W. W. Jamieson,Wave Forces on Square Caissons, Coastal Engineering,15th International Conference on Coastal Engineering, 1976

[5] Zhao Zidan,Lu Junliang, Small amplitude wave action on rectangular vertical pile(In Chinese), Acta Oceanologica Sinica (J), 1980-03

[6] Huang Hening, Analytic solution of large rectangular cylinder (In Chinese), Applied Mathematics and Mechanics(J),1988-07

[7] Huang Hening, Inertia force coefficient of arbitrary heading incident wave on small rectangular cylinder (In Chinese), Coastal Engineering(J),1990-2

[8] Roland Schinzinger, Patricio A. A. Laura, Conformal Mapping: Methods and Applications, Dover Publications,2003

[9] L. V. Kantorovich and V. I. Krylov, Approximate Methods of Higher Analysis, translated by C. D. Benster, P. Noordhoff Ltd,1958

[10] Abramowitz, M. and Stegun, I. A. (Eds.). Handbook of Mathematical Functions with Formulas, Graphs, and Mathematical Tables, 9th printing. New York: Dover, 1972.

[11] Bender, C., S. Orszag. Advanced Mathematical Methods for Scientists and Engineers. NY: McGraw-Hill, 1978

[12] Hung Cheng ,Advanced Analytic Methods in Applied Mathematics, Science, and Engineering,LuBan Press,2006

\footnotetext{
About Author :
}

Fan Huiqu, PhD student of China Ship Scientific Research Center, PRC. 